\title{
Fluorescent Excitation of Spectral Lines In Planetary Nebulae
}

\author{
Guo Xin Chen and Anil K. Pradhan \\ Department of Astronomy, The Ohio State University, Columbus, OH 43210
}

\begin{abstract}
Fluorescent excitation of spectral lines is demonstrated as a function of temperatureluminosity and the distance of the emitting region from the central stars of planetary nebulae. The electron densities and temperatures are determined, and the method is exemplified through a detailed analysis of spectral observations of a high excitation PN, NGC 6741, observed by Hyung and Aller (1997). Fluorescence should also be important in the determination of element abundances. It is suggested that the method could be generally applied to determine or constrain the luminosity and the region of spectral emission in other intensively radiative sources such as novae, supernovae, and active galactic nuclei.
\end{abstract}

Subject headings: planetary nebulae: general - H II regions — atomic data — line: formation radiation mechanisms: thermal — radiative transfer

\section{INTRODUCTION}

In a classic paper (Seaton 1978) discussed the excitation of nebular emission lines by resonant scattering of radiation from the central stars, and found that in addition to electron scattering and recombination, absorption of radiation should be taken into account in the analysis of O III lines and consequent determination of oxygen abundance. The high intensity of the continuum radiation from planetary nebulae, and other sources, are likely candidates for such fluorescent excitation (hereafter FLE) of spectral lines, provided the atomic structure of the given ionic species (such as O III) leads itself to resonant excitation from the ground state, followed by cascades into upper levels of observed transitions. Given that FLE is operative, it follows that the intensities of spectral lines (or line ratios) would also be dependent on the radiation temperature-luminosity of the source and the region of the dominant abundance of the ionic species, i.e. the distance from the source, via a geometrical dilution factor. It was shown by Lucy (1995) that the nickel overabundance problem in a variety of gaseous nebulae may be addressed by taking into account the FLE of [Ni II] optical lines by the background stellar UV continuum. Following Lucy (1995), Bautista et al
(1996) and Bautista and Pradhan (1998) investigated FLE in [Fe II] optical transitions, but found it to be less effective in line formation than [Ni II] owing to differences in atomic structure and associated transition probabilities.

In the present Letter we explore FLE in a more general manner, using optical emission lines from a moderately high ionization state of iron, Fe VI, observed in several planetary nebulae in extensive observational studies by Hyung and Aller (e.g. 1995,1996,1997,1998). In particular we focus on NGC 6741 (Hyung and Aller 1997) where we find clear evidence of FLE. It is shown that the observed [Fe VI] line ratios can only be explained with FLE, and yield electron densities lower than those derived from other less ionized species such as the well known [O II] and [S II] fine structure doublets (Osterbrock 1989). The inferred distances of the $[\mathrm{Fe} \mathrm{VI}]$ emission region from the central stars is consistent with the $\mathrm{He}^{2+}$ zone (Seaton (1978)), within the inner radius of the nebulosity. Possible implications of this work are discussed in relation to other sources, such as novae and AGN, where FLE of spectral lines should be important in the determination of densities,temperatures, abundances, and the spatial extent of emission regions. It is shown that surface contour plots of line ratios as double-valued 
function of radiation temperature of the source, and the dilution factor, may serve to constrain or determine both quantities from theoretical calculations and observations.

\section{FLUORESCENT EXCITATION OF Fe VI}

Given an external radiation field, atomic FLE can be effective for excitation from the ground state and subsequent cascades to levels via dipole allowed transitions. The $\Delta S=0$ transitions, where $(2 \mathrm{~S}+1)$ is the spin multiplicity, are generally more efficient than the intercombination $\Delta S \neq 0$ cascade transitions, since the former usually have orders of magnitude higher transition probabilities. A schematic representation of the energy level structure of Fe VI with FLE is given in Fig. 1. The dominant UV excitations from the even parity ground LS term $3 d^{3}\left({ }^{4} F_{J}\right)$ and fine structure levels $J=3 / 2,5 / 2,7 / 2,9 / 2$ to the odd parity levels of the terms of the excited configuration $3 d^{2} 4 p\left({ }^{4} G_{J}^{o},{ }^{4} F_{J}^{o},{ }^{4} D_{J}^{o}\right)$ are strong dipole allowed transitions at $3.08-3.15$ Rydbergs in the far UV range 289 - $296 \stackrel{\circ}{A}$.

A collisional-radiative model with FLE involving 80 fine structure levels for Fe VI is employed to calculate level populations, $N_{i}$, relative to the ground level. The emitted flux per ion for transition $j \rightarrow i$, or the emissivity $\epsilon_{j i}\left(\operatorname{ergs} \mathrm{cm}^{-3} \mathrm{~s}^{-1}\right)$, is given by, $\epsilon_{j i}=N_{j} A_{j i} h \nu_{i j}$. In matrix notation the coupled equations of statistical equilibrium for the optically thin case can be expressed as: $\mathbf{C}=\mathbf{N}_{e} \mathbf{Q}+\mathbf{A}+\mathbf{J B}$, where $N_{e}$ is the electron density, $\mathbf{Q}, \mathbf{A}$, and $\mathbf{B}$ are the collisional excitation and the Einstein spontaneous and stimulated emission rate matrices, and $\mathbf{J}$ represents the photon density from a continuum radiation field. The matrix elements of the total excitation rate $\mathrm{C}_{i j}$ are then expressed as:

$$
\begin{gathered}
C_{i j}=q_{i j} N_{e}+J_{i j} B_{i j} \quad(j>i), \\
C_{i j}=q_{i j} N_{e}+A_{i j}+J_{i j} B_{i j} \quad(j<i),
\end{gathered}
$$

where $J_{i j}$ are the mean intensities of the continuum at the frequency for transition $i \rightarrow j$. For example, for a thermal source at effective temperature $T_{e f f}$, the monochromatic flux at the photosphere $F_{\nu}$ is given by the Planck function

$$
J_{i j}=W F_{\nu}=W \frac{8 \pi h \nu^{3}}{c^{2}} \frac{1}{e^{h \nu / k T_{e f f}}-1}
$$

where $W=\frac{1}{4}\left(\frac{R_{*}}{r}\right)^{2}=1.27 \times 10^{-16}\left(\frac{R_{*} / R_{\odot}}{r / p c}\right)^{2}$ is the geometrical dilution factor; $R_{*}$ and $\mathrm{r}$ are the radius of the photosphere and the distance between the star and the emission region respectively.

All required atomic data have been recently computed by the authors under the Iron Project (Hummer et. al. 1993). This includes collision strengths for 3,160 transitions among the $80 \mathrm{Fe} \mathrm{VI}$ levels (Chen and Pradhan 1999a,b), and transition probabilities for 867 allowed electric dipole (E1) and 1,230 forbidden electric quadrupole and magnetic dipole (E2,M1) transitions (Chen and Pradhan 1999c). While the E2,M1 A-values agree reasonably well with the earlier work on [Fe VI] by Nussbaumer and Storey (1978), the present collision strength data differs considerably from theirs owing to resonance effects (Nussbaumer and Storey (1978) did not consider the FLE mechanism for [Fe VI] line formation). A detailed discussion is given by Chen and Pradhan (1999c), which also presents more extensive results on the computed Fe VI line emissivity ratios than considered in this Letter).

\section{PLANETARY NEBULA NGC 6741}

Observations of this high excitation nebula by Aller et al (1985) and Hyung and Aller (1997) show several optical [Fe VI] lines in the spectrum from the multiplet $3 d^{3}\left({ }^{4} F-{ }^{4} P\right)$ at 5177, 5278, $5336,5425,5485,5632$ and $5678 \AA$ and from the $\left({ }^{4} F-{ }^{2} G\right)$ at 4973 and $5147 \stackrel{\circ}{A}$. The basic observational parameters, in particular the inner and the outer radii needed to estimate the distance from the central star and the dilution factor, are described in these works, and their diagnostic diagrams based on the spectra of a number of ions give $T_{e}=12500 \mathrm{~K}, N_{e}=6300 \mathrm{~cm}^{-3}$, and a stellar $T_{\text {eff }}=140,000 \mathrm{~K}$. As the ionization potentials of Fe V and Fe VI are $75.5 \mathrm{eV}$ and $100 \mathrm{eV}$ respectively, compared to that of He II at $54.4 \mathrm{eV}$, Fe VI emission should stem from the fully ionized $\mathrm{He}^{2+}$ zone, and within the inner radius, i.e. $\mathrm{r}(\mathrm{Fe} \mathrm{VI})$ $\leq r_{i n}$. With these parameters we obtain the dilution factor to be $\mathrm{W}=10^{-14}$; the dominant $[\mathrm{Fe} \mathrm{VI}]$ 
emission region could be up to a factor of 3 closer to the star, with $\mathrm{W}$ up to $10^{-13}$, without large variations in the results obtained.

Fig. 2 presents 4 [Fe VI] line ratios as a function of several parameters, in particular with and without FLE. In all cases the FLE $=0$ curve fails to correlate with the observed line ratios, and shows no dependence on $\mathrm{N}_{e}$ (an unphysical result), whereas with FLE we obtain a consistent $N_{e} \approx$ $1000-2000 \mathrm{~cm}^{-3}$, suitable for the high ionization [Fe VI] zone. The derived $\mathrm{N}_{e}$ is somewhat lower than the $\mathrm{N}_{e}$ range $2000-6300 \mathrm{~cm}^{-3}$ obtained from several ionic spectra (including [O II] and [S II]) by Hyung and Aller (1997). Exactly similar results are obtained for 3 other line ratios of [Fe VI]; those have been omitted from Fig. 2 for brevity. The total observational uncertainties are $10 \%, 16 \%, 20 \%$ and $23 \%$ for the $5485,5336,5177$ and 5425 line ratios (with respect to the $5147 \AA$ line), respectively (Hyung and Aller 1997). However, an indication of the overall uncertainties may be obtained from the 8 th line ratio, $4973 / 5147$, which is independent of both $\mathrm{T}_{e}$ and $\mathrm{N}_{e}$ since both lines have the same upper level, and which therefore depends only on the ratio of the A-values; the observed value of 1.048 agrees closely with the theoretical value of 0.964 . Whereas the combined observational and theoretical uncertainties for any one line ratio can be significant, all measured line ratios yield a remarkably consistent $\mathrm{N}_{e}([\mathrm{Fe} \mathrm{VI}])$ and substantiate the spectral model with FLE.

\section{TEMPERATURE-LUMINOSITY AND THE EMISSION REGION}

Having determined the dependence of [Fe VI] line intensities with FLE on local quantities, the electron density and temperature in the emission region $\left\{\mathrm{N}_{e}, \mathrm{~T}_{e}\right\}$ and related photon flux, we next examine the dependence on the macroscopic parameters, the stellar radiation temperature and the distance indicated by the dilution factor $\left\{\mathrm{T}_{\text {eff }}, \mathrm{W}(\mathrm{r})\right\}$. Fig. 3 shows the variation in two line ratios, with $\mathrm{W}(\mathrm{r})$, at three $\mathrm{T}_{\text {eff }}$ generally corresponding to the range of stellar temperatures of the central stars of PN. It is seen that the correlation involving the photon density and the source radiation temperature is fairly well defined, analogous to the dependence on electron density $\mathrm{N}_{e}$ in a characteristic range of electron temperatures
$\mathrm{T}_{e}$. It follows that the loci of the line ratios may be associated with photon and electron densities in the emission region and may be parametrized. A given range of dilution factors, indicating the likely distances of the emission region from the source, and the radiation intensity at the source, may therefore be constrained to a most probable set of $\left\{\mathrm{T}_{\text {eff }}, \mathrm{W}(\mathrm{r})\right\}$.

Fig. 4 illustrates a three-dimensional $\mathrm{R}\left(\mathrm{T}_{\text {eff }}\right.$, $\mathrm{W}(\mathrm{r}))$ surface, intersected by a plane defined by the observed line ratio $\mathrm{R}=\mathrm{R}(\mathrm{obs})=0.460$, along a contour of $\left\{\mathrm{T}_{e f f}, \mathrm{~W}(\mathrm{r})\right\}$. The electron density and temperature are $\mathrm{N}_{e}=2000 \mathrm{~cm}^{-3}$ and $\mathrm{T}_{e}=12,000$ $\mathrm{K}$, as derived from the line ratios in Fig. 2. It is seen that while the effective source temperature and the distance of the emission region are not uniquely defined as a 1-1 correspondence, they are constrained within a contour by $\mathrm{R}(\mathrm{obs})$. In case of a source with a higher value of $\mathrm{R}(\mathrm{obs})$, the contour $\left\{\mathrm{T}_{\text {eff }}, \mathrm{W}(\mathrm{r})\right\}$ may be more stringently described.

\section{DISCUSSION AND CONCLUSION}

Fluorescence in selected atomic species may be included in spectral models to constrain the parameter space $\left\{\mathrm{N}_{e}, \mathrm{~T}_{e}, \mathrm{~T}_{e f f}, \mathrm{~W}(\mathrm{r})\right\}$ from theoretical and observed line ratios. The main parts of such an analysis are: (i) the determination of $\mathrm{N}_{e}$ and $\mathrm{T}_{e}$ from line ratios with and without FLE (Fig. 2), (ii) examination of the luminosity function at $\mathrm{T}_{\text {eff }}$ vs. $\mathrm{W}(\mathrm{r})$ (Fig. 3), and (iii) calculation of possible $\mathrm{R}\left(\mathrm{T}_{\text {eff }}, \mathrm{W}(\mathrm{r})\right)$ contours (Fig. 4). For sufficient precision it is necessary to study several line ratios of the given ion, and to include all relevant atomic transitions in the model. It is also required that the radiative and collisional atomic data be know a priori with high accuracy. Both of these criteria are are now feasible with increasingly extensive and high-resolution space and ground spectral observations, and large-scale theoretical calculations such as under the Iron Project (Hummer et. al. 1993).

We have analyzed the spectra of a few other PN: NGC 6886, Hubble 12, NGC 2440 (Hyung et al 1995; Hyung and Aller 1996, 1998). These more detailed results will be presented in a companion publication (Chen and Pradhan 1999c). Interestingly, extragalactic spectral observations of [Fe VI] lines at 5147 and $5177 \AA$ have also been reported by Meatheringham and Dopita (1991) from PN 
SMP22 in the Small Magellanic Cloud. As with the galactic PN, the observed 5177/5147 line ratio of 0.83 in SMC SMP22 does not correspond to realistic $\left(\mathrm{N}_{e}, \mathrm{~T}_{e}\right)$ without FLE. An optimum parameter fit with FLE however yields $\mathrm{N}_{e}([\mathrm{Fe} \mathrm{VI}])$ $\approx 1000 \mathrm{~cm}^{-3}$, at $\mathrm{T}_{e}=20,000 \mathrm{~K}$ (Meatheringham and Dopita report $\left.\mathrm{T}_{e}\left(\left[\begin{array}{ll}\mathrm{O} & \mathrm{III}\end{array}\right]\right)=26,600 \mathrm{~K}\right)$, and $\mathrm{W}(\mathrm{r})=10^{-13}, \mathrm{~T}_{\text {eff }}=150,000 \mathrm{~K}$. Strictly speaking $\mathrm{W}(\mathrm{r}) \longrightarrow \mathrm{R}_{*} / \mathrm{r}$; i.e. it yields the ratio of the stellar radius to the distance of the emission region. It is also important to note that the determination of total and fractional element abundances could be highly uncertain if the effect of FLE on spectral formation is not ascertained accurately.

The proposed approach could possibly be employed to study variable central luminosity sources such as novae and AGN. With variations in electron density and temperature, and the spatial extent of the emission region, a given line ratio may exhibit large variations with temperatureluminosity, as illustrated in Figs. 3 and 4 . It may also be noted that the method is not predicated on the assumption of coronal or photoionization equilibrium, generally assumed in modeling nebulae and AGN. However, further constraints may be sought from calculations such as coronal, and/or photoionization, models in ionization equilibrium that determine the ionization fraction of an element vs. r. Conversely, determination of peak abundance fractions from models may be verified through direct spectroscopic analysis of FLE dominated emission from an ion, as described herein. For objects where the nebulosity is observed and spatially defined, determination of $\mathrm{T}_{\text {eff }}$ (temperature-luminosity) of the source may yield independent distance estimates via triangulation.

In non-ionization equilibrium the spectral modeling might entail level-specific ionization / recombination involving metastable populations (work in progress). Non-thermal specific luminosity or a mean photon intensity function vs. frequency should be applied in real applications, e.g. in the synchrotron continuum pumping in Crab nebula (Davidson \& Fesen 1985; Lucy 1995), or a powerlaw spectrum in AGN. Future FLE work in intense radiation background sources involves the analysis of the symbiotic nova RR Tel (McKenna et al 1997) and the star V 1016 Cygni (a possible single $\mathrm{PN}$ in formation or a symbiotic nova) (Ahern et al 1977), where many [Fe VI] emission lines have been observed, the spectra of 'coronal' iron ions Fe XVIII - XVII in narrow or coronal line region (NLR,CLR) of AGN, and [Ni II] and [Co II] lines in nebular remnants of supernovae type II (e.g. 1987A) and Ia.

Given that spectral formation from an atomic species is localized with respect to the radiative source, and subject to FLE, variation in spectral intensities with photon density should enable qualitative and quantitative constraints on the nature of the source and the emission region. To that end, the radiation field could be thermal or nonthermal, and the geometrical dilution with distance to the emission region could be suitably parameterized, including local variables such as electron density and temperature (radiative transfer effects would be important at significant optical depths).

Finally we note that in addition to demonstration of (a) FLE, and (b) regular intensity variations in line emitting regions, studies of (a) and (b) may resolve discrepancies in element abundance determinations from recombination lines and using collisionally excited lines without FLE (Seaton 1978).

This work was partially supported by the NSF (AST-9870089) and NASA (NAG5-8423).

\section{REFERENCES}

Ahern, F.J., FitzGerald, M.F., Marsh, K.A., \& Purton, C.R., 1977, A\&A, 58, 35

Aller, L.H., Keys, C.D., \& Czyzak, S.J., 1985, ApJ, 296, 493

Bautista, M.A., Peng, J.F., \& Pradhan, A.K., 1996, ApJ 460, 372

Bautista, M.A., \& Pradhan, A.K., 1998, ApJ 492, 650

Chen, G.X., \& Pradhan, A.K., 1999a, Journal of Physics B32, 1809 1999b A\&AS 136, 395

Chen, G.X., \& Pradhan, A.K., 1999c, MNRAS, in preparation

Davidson, K., \& Fesen, R.A. 1985, ARA\&A, 23, 119 
Hummer D.G., Berrington K.A., Eissner W., Pradhan A.K, Saraph H.E., \& Tully J.A., 1993, A\&A, 279, 298

Hyung, S., Keyes, C.D. \& Aller, L.H., 1995, MNRAS, 272, 49

Hyung, S., \& Aller, L.H., 1996, MNRAS, 278, 551

Hyung, S., \& Aller, L.H., 1997, MNRAS, 292, 71

Hyung, S., \& Aller, L.H., 1998, Pub. Astron. Soc. Pacific, 110, 466

Lucy, L.B., 1995, A\&A, 294, 555

McKenna, F.C.,Keenan, F.P., Hambly, N.C., Prieto, A., Rolleston, W.R.J., Aller, L.H., \& Feibelman, W.A., 1997, ApJS, 109, 225

Meatheringham, S.J., \& Dopita, M.A., 1991, ApJS, 75, 407

Nussbaumer, H., \& Storey, P.J., 1978, A\&A, 70, 37

Osterbrock, D.E., 1989, Astrophysics of Gaseous Nebulae and Active Galactic Nuclei (New York: University Science Books)

Seaton, M.J., 1978, MNRAS, 139, 129

\section{Figure Captions}

Fig. 1.- Schematic diagram of fluorescent excitation (FLE) of [Fe VI] optical emission lines. The full radiative-collisional model includes 80 fine structure levels.

Fig. 2.- Diagnostic line ratios for PN NGC 6741 Hyung and Aller (1997), with and without FLE of Fe VI. The $\mathrm{W}=0$ (no FLE) does not correspond to any observed line ratio.

Fig. 3.- Variation of line ratio with geometrical dilution factor $\mathrm{W}(\mathrm{r})$, and the effective radiation temperature $\mathrm{T}_{\text {eff }}$, indicating the distances and localization of the emission region.

Fig. 4.- Spectral intensity variation vs. $\left\{\mathrm{T}_{\text {eff }}\right.$, $\mathrm{W}(\mathrm{r})\}$. Intersection of the plane defined by the observed line ratio in NGC 6741 with this surface describes a contour of possible set of $\left\{\mathrm{T}_{e f f}, \mathrm{~W}(\mathrm{r})\right\}$. The electron density and temperature $\left\{\mathrm{N}_{e}, \mathrm{~T}_{e}\right\}$ are determined as in Fig. 2.

This 2-column preprint was prepared with the AAS IATEX macros v5.0. 
Fluorescent Excitation of $\mathrm{Fe} \mathrm{VI}$

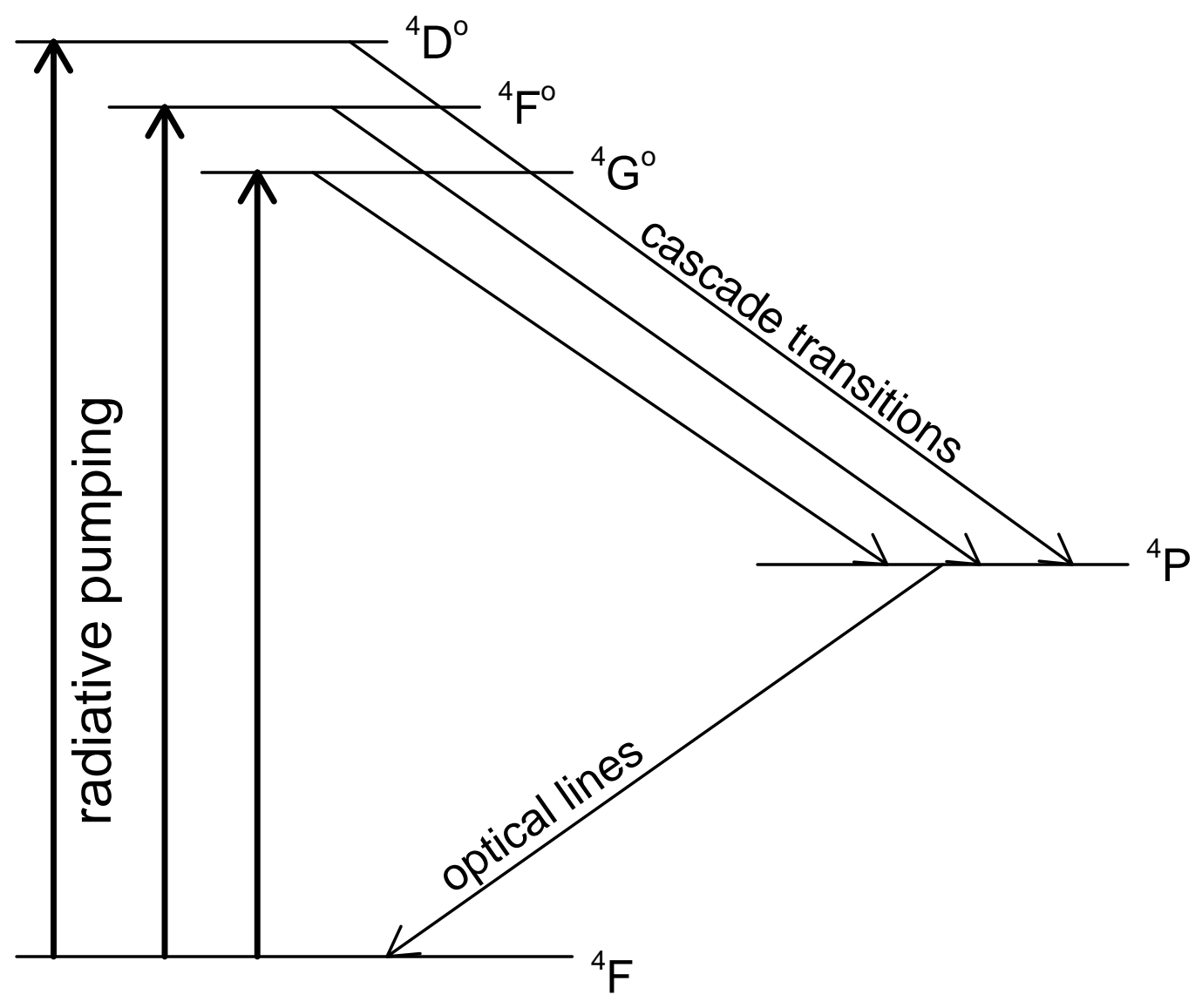



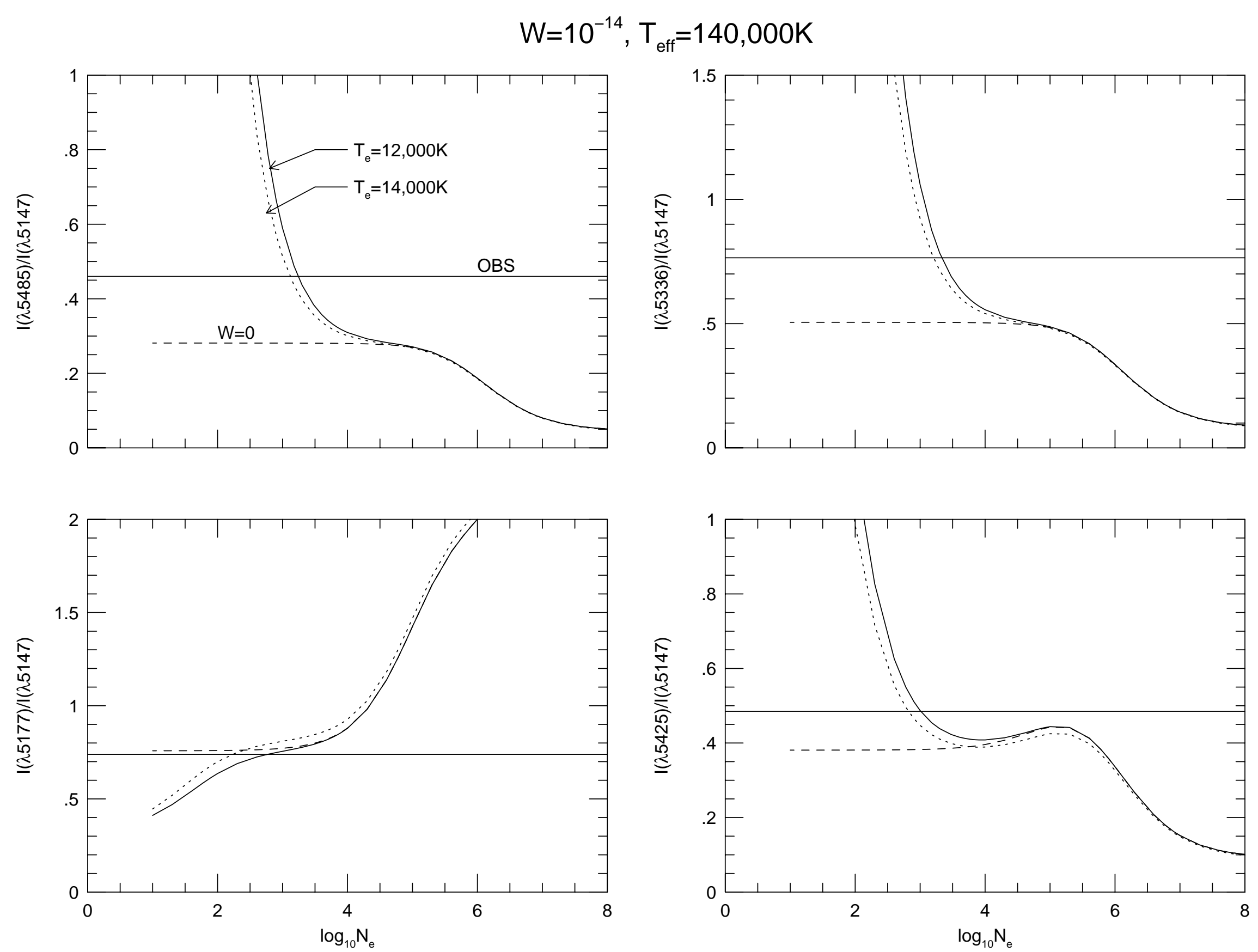

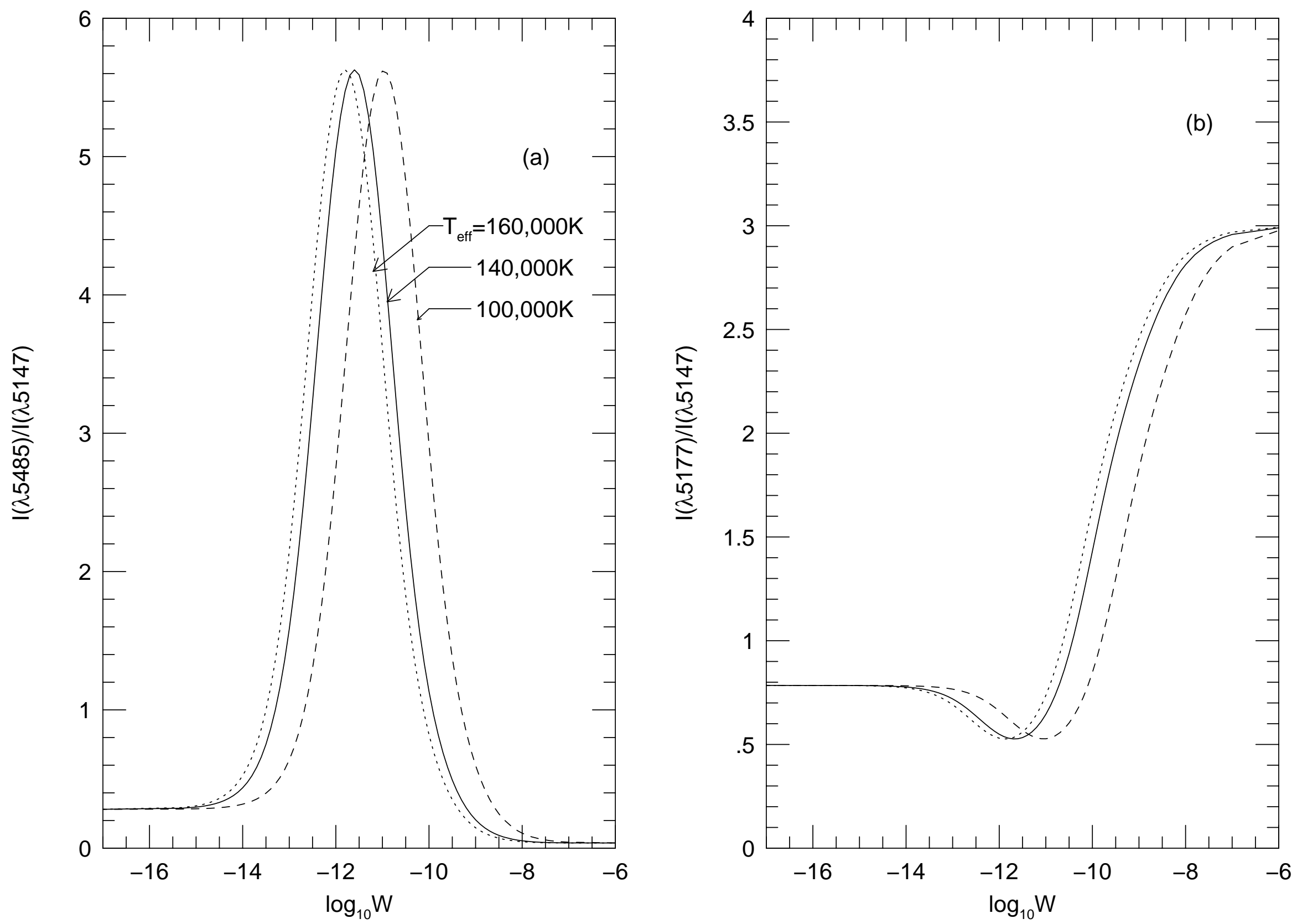


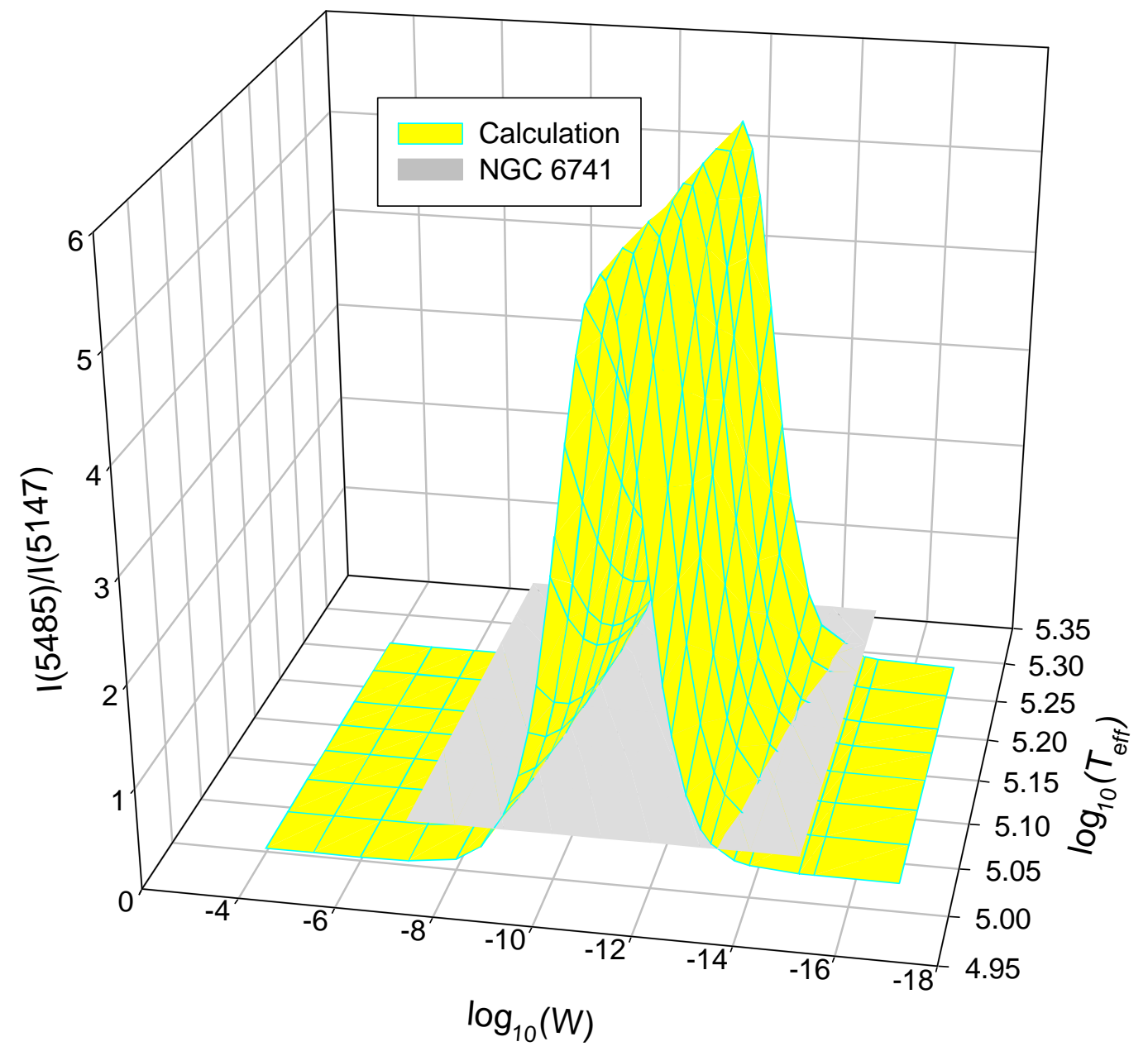

